\title{
Contrast adaptation dissociates different measures of luminous efficiency
}

\author{
Michael A. Webster and J. D. Mollon \\ Department of Experimental Psychology, University of Cambridge, Downing Street, Cambridge CB2 3EB, UK
}

Received August 26, 1992; accepted October 7, 1992; revised manuscript received December 18, 1992

\begin{abstract}
We compared how contrast adaptation influences three alternative measures of luminous efficiency. Subjects judged the lightness, the flicker, or the motion of chromatic sine-wave gratings. Adaptation to gratings with correlated luminance and chromatic contrast strongly biases lightness matches and moderately biases minimum-motion settings for gratings that are counterphased at $1 \mathrm{~Hz}$, but it has little effect on motion or flicker settings for gratings that are counterphased at $15 \mathrm{~Hz}$. These results suggest that different measures of equiluminance tap neural pathways that can have different spectral sensitivities. At low temporal frequencies both perceived lightness and minimum-motion settings appear to depend on channels that do not represent luminance and color independently.
\end{abstract}

\section{INTRODUCTION}

Light is initially encoded in the human visual system by three separate classes of cone receptor, but subsequently the signals from different cone types are combined, either with the same sign to form mechanisms that are sensitive to luminance or with opposite signs to form mechanisms that are sensitive to color. ${ }^{1,2}$ These transformations may occur in parallel within a variety of different subsystems that are designed to extract different properties of the retinal image, such as movement, form, and depth.,3,4 Thus the triplet of retinal cone signals may be recombined with different weights and signs within different subsystems, and different perceptual tasks may depend on markedly different representations of the luminance and chromatic contrasts in the image. We measured the spectral sensitivity underlying three alternative perceptual judgments - of lightness, flicker, and minimum motionand compared how these alternative judgments are affected by adaptation to contrast, i.e., adaptation to patterns whose color and luminance are modulated around a fixed average color and luminance. Contrast adaptation changes sensitivity to luminance and chromatic stimuli, ${ }^{5-7}$ and we asked whether alternative measures of these sensitivity changes reflect different weightings of the cone signals.

Using stimulus conditions and experimental procedures that were matched as closely as possible, we compared three alternative criteria for equating the luminous efficiencies of two colors that were presented as components of a heterochromatic sine-wave grating. The gratings were counterphased at 1 or $15 \mathrm{~Hz}$.

(a) Perceived lightness $(1 \mathrm{~Hz})$. At low temporal frequencies the interleaved colors of the grating can be resolved, and they can be matched for lightness ${ }^{8}$ by varying their relative radiances.

(b) Flicker photometry $(15 \mathrm{~Hz}){ }^{9}$ When two colors are alternated rapidly in time, sensitivity to the color difference is reduced, yet a luminance difference between the colors is still visible as flicker. The two colors are defined as equiluminant when perceived flicker is minimized. (c) Minimum motion ( 1 and $15 \mathrm{~Hz}$ ). ${ }^{10}$ Luminance differences in a chromatic test grating can combine with an added achromatic grating (superposed in spatial and temporal quadrature phase with the test grating ${ }^{11}$ ) to yield a drifting luminance grating (Plate I). The direction of motion depends on which component color in the chromatic grating has the higher luminosity. Equiluminance can therefore be defined as the relative luminosities of the component colors at which the direction of motion reverses.

Flicker photometry and minimum motion yield similar estimates of the relative luminous efficiency of different spectral stimuli. ${ }^{12}$ These estimates of sensation luminance ${ }^{13}$ are, for individual observers, the equivalent of the sensitivity standardized in 1924 by the $V(\lambda)$ weighting function of the CIE. ${ }^{14}$ In contrast, for many subjects, heterochromatic brightness matches yield spectral sensitivities that differ from flicker-photometric estimates and are nonadditive. ${ }^{15-17}$ This result has suggested that perceived brightness depends on the combined outputs of both luminance and chromatic channels. ${ }^{16,18}$

Adaptation to a stimulus that covaries in luminance and chromaticity can induce a lightness difference between two chromaticities that were matched for lightness before adaptation: the appearance of the equiluminant plane is effectively tilted away from the axis in color-luminance space of the adapting modulation (Fig. 1). ${ }^{7,19}$ For example, after adaptation to a contrast defined by modulations between light red and dark green, an equiluminant red appears darker, whereas an equiluminant green appears lighter. These lightness biases reflect a selective loss in sensitivity to the adapting axis and occur without significant change in the average perceived lightness or color of the test stimuli. Thus they cannot be accounted for by the average response changes that are conventionally studied in color adaptation ${ }^{20}$ and instead depend on changes in sensitivity to contrast- to how luminance and color are varying around the average. However, the form of these contrast changes is inconsistent with an adaptation effect that occurs independently within separate luminance and chromatic channels: gain changes in such 


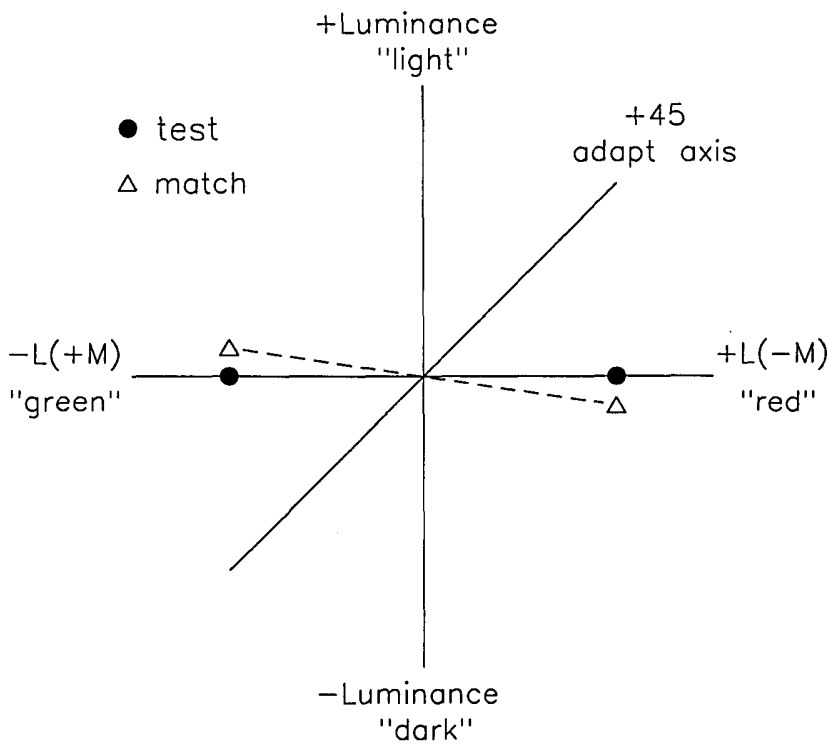

(a)

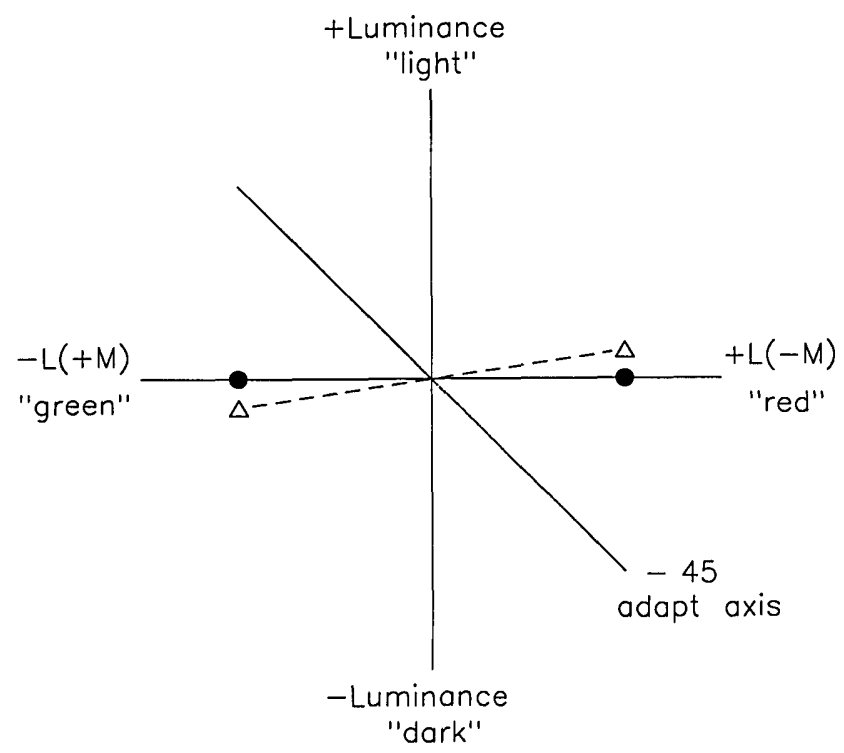

(b)

Fig. 1. (a) Plane in color space defined by the luminance axis and a chromatic axis that represents differences in $L-$ and M-cone signals and varies from greenish to reddish. Along the 45-deg axis luminance and $+\mathrm{L}(-\mathrm{M})$ chromatic signals are positively correlated. Adaptation to this axis can bias lightnesses along the L-M chromatic axis away from the adapting axis, so that an originally equiluminant red and green are now matched to a darker red and a lighter green. ${ }^{7}$ (b) Along the -45-deg axis the same luminance and $+\mathrm{L}(-\mathrm{M})$ chromatic signals are negatively correlated. Adaptation to this axis can produce the opposite biases along the $\mathrm{L}-\mathrm{M}$ axis.

channels could change sensitivity to luminance and chromatic contrast but could not induce a luminosity difference between two stimuli that isolate chromatic channels. The observed biases suggest instead that perceived lightness depends on channels that can be selectively tuned to different combinations of luminance and chromatic contrast and that the representation of lightness varies with the state of adaptation, presumably because adaptation selectively changes the distribution of responses within these channels. Here we examine whether these biases reflect a general change in the spectral sensitivity of the visual system by testing whether adaptation induces similar biases in flicker and minimum-motion estimates of sensitivity.

\section{METHOD}

Stimuli were displayed on a Sony GVM1400QM monitor controlled by a Cambridge Research Systems graphics board (which allowed radiances on the monitor to be controlled with an accuracy of 14 bits/gun). Luminances of the three guns were linearized through lookup tables. Subjects (the two authors) viewed the display monocularly with natural pupils from a distance of $1.5 \mathrm{~m}$.

The stimuli were presented in a 2-deg-square field with central fixation cross. Narrow black borders delimited the field from a surround (4.8 deg vertical $\times 9.6 \mathrm{deg}$ horizontal) of the same average luminance $\left(27.5 \mathrm{~cd} / \mathrm{m}^{2}\right)$ and chromaticity (equivalent to Illuminant $\mathrm{C}$ ). Test stimuli were 1.5 cycles per degree (c/deg) heterochromatic sinewave gratings, counterphased at 1 or $15 \mathrm{~Hz}$ and oriented horizontally. The chromatic variations defining the gratings were empirically chosen (as in Ref. 21) either to modulate the signals in the short-wavelength cones while holding signals in the long- and medium-wavelength cones constant (S) or to modulate the ratio of signals in the longand medium-wavelength cones while holding signals in the short-wavelength cones constant (L-M). Many properties of color vision appear to be organized in terms of these two cardinal chromatic dimensions. ${ }^{6,22,23}$ For the L-M test patterns, chromatic contrast was fixed at $50 \times$ the contrast threshold for detecting the $1-\mathrm{Hz}$ counterphasing L-M grating, whereas for the S patterns it was fixed at $30 \times$ threshold. The $15-\mathrm{Hz}$ patterns had the same physical chromatic contrast as the $1-\mathrm{Hz}$ gratings but much lower contrasts in terms of multiples of threshold. (These values were chosen to be close to the maximum contrasts possible for the test and adapting stimuli for our display.) The 1-Hz thresholds were based on measurements for observer MW. The same contrasts were used for observer JM. The chromaticity coordinates corresponding to these chromatic contrasts are listed in Table 1.

For lightness or flicker judgments, only a single counterphasing test grating was presented, whereas for minimummotion judgments a second counterphasing luminance grating was added $90 \mathrm{deg}$ out of phase with the chromatic test grating in both space and time (see Plate I). The Michelson contrast of the added luminance grating was either 0.135 (when combined with the L-M test gratings) or 0.10 (when combined with the $\mathrm{S}$ test gratings). The modulation of the chromatic test gratings was in sine phase with the start of the test trial and in spatial cosine phase with the center of the display. For observer MW the added luminance grating in the minimum-motion task always began in cosine phase. However, for observer JM we added the control of randomly varying on each trial the starting phase of the luminance grating between plus or minus cosine phase, so that the direction of motion would be uncorrelated with the sign of the luminance contrast in the chromatic test grating.

In the case of motion or lightness judgments, changes in the sign of the luminance mismatch (e.g., green luminance greater versus red luminance greater) result in qualita- 
Table 1. Chromaticity Coordinates of Test and Adaptation Gratings Whose Chromatic Contrasts Modulate Either Signals in the S Cones or the Ratio of Signals in the L and M Cones ${ }^{a}$

CIE $1931(x, y)$

MacLeod-Boynton $(r, b)$

\begin{tabular}{ccc}
$+\mathrm{L}(-\mathrm{M})$ & $\mathrm{L}-\mathrm{M}$ mean & $-\mathrm{L}(+\mathrm{M})$ \\
\hline$(0.351,0.301)$ & $(0.310,0.316)$ & $(0.264,0.333)$ \\
$(0.686,0.0178)$ & $(0.6565,0.01825)$ & $(0.627,0.0187)$ \\
& & \\
$+\mathrm{S}$ & $\mathrm{S}$ mean & $-\mathrm{S}$ \\
\hline$(0.280, .0249)$ & $(0.310,0.316)$ & $(0.362,0.429)$ \\
$(0.655,0.0289)$ & $(0.6565,0.01825)$ & $(0.658,0.0076)$
\end{tabular}

CIE $1931(x, y)$

MacLeod-Boynton $(r, b)$

$(0.655,0.0289)$

$(0.6565,0.01825)$

aThe coordinates express the chromaticities of the two component colors in the grating in terms of either the 1931 CIE chromaticity diagram or the MacLeod-Boynton chromaticity diagram, ${ }^{24}$ whose $b$ and $r$ axes more directly represent the $\mathrm{S}$ and L-M chromatic variations. Both chromatic contrasts differed in both $b$ and $r$ because the empirically defined $\mathrm{S}$ and L-M axes differed slightly from those of the standard observer.

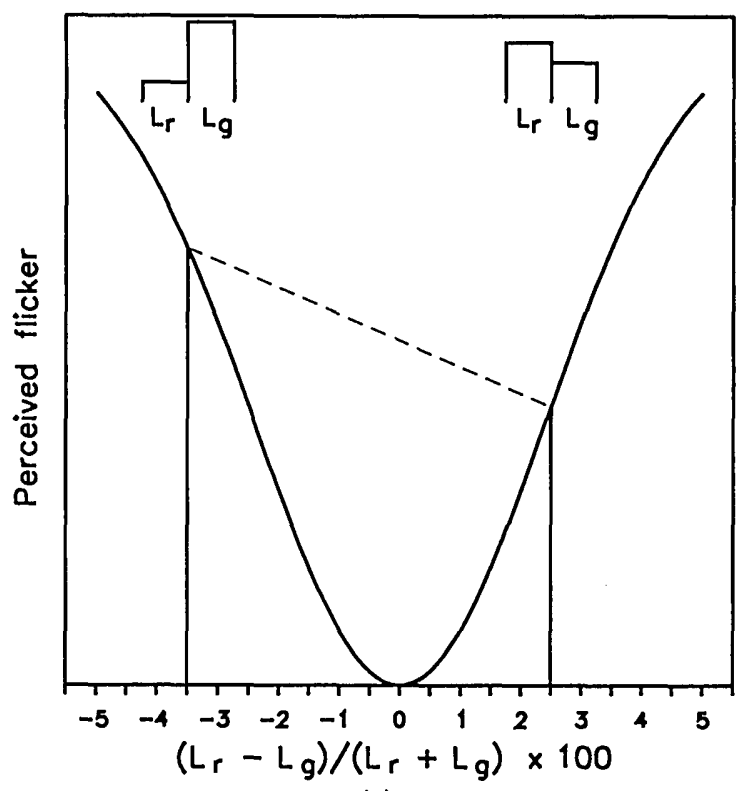

(a)

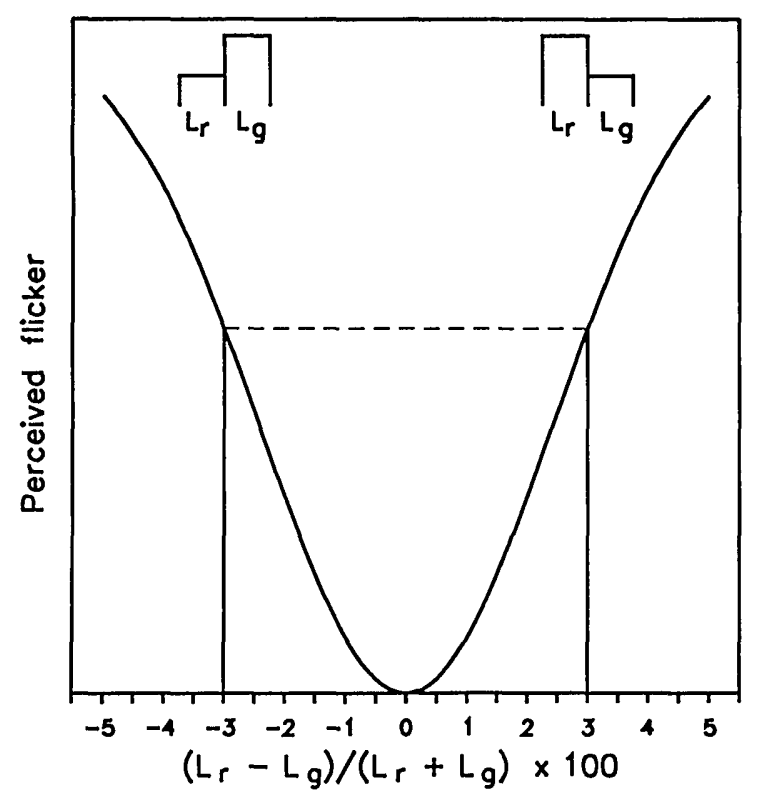

(b)

tively different sensations (up versus down for motion, or green lighter versus red lighter for lightness). Thus the subject's response indicates how luminance contrast must be altered to approach the equiluminant point. However, for flicker these opposite luminance mismatches are difficult to distinguish. Subjects instead base their flicker

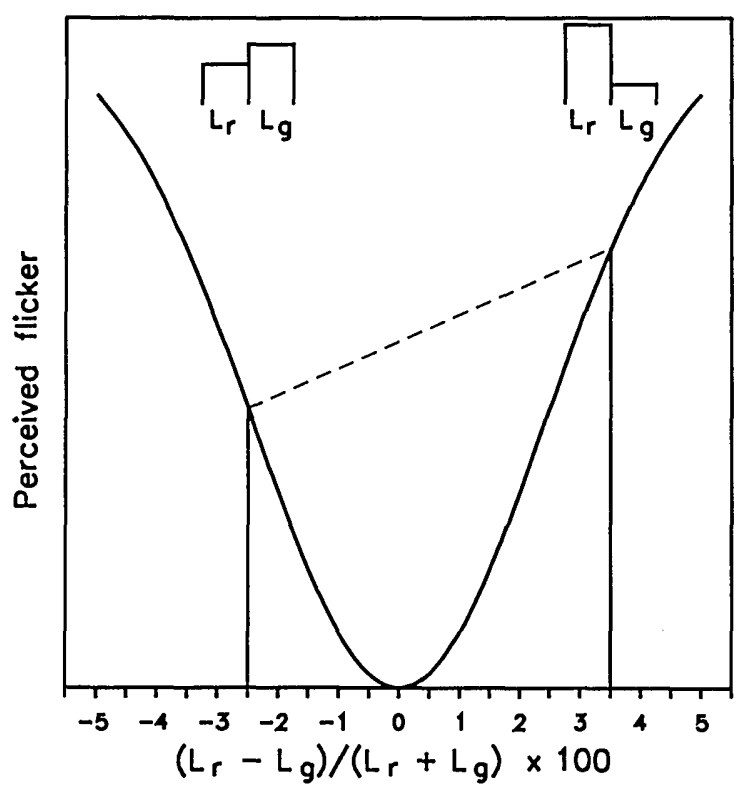

(c)

Fig. 2. Hypothetical stimulus and response in a split-field flicker discrimination. Luminance contrast is introduced between the red and green bars of a red-green chromatic grating. In one half-field (e.g., top) of the display, red bars have a higher luminance $\left(\mathrm{L}_{\mathrm{r}}\right)$, whereas in the other half-field (e.g., bottom) green bars have a higher luminance $\left(\mathrm{L}_{\mathrm{g}}\right)$. The grating is counterphased, and thus at each spatial position the bars alternate between bright red and dark green in one half-field or between bright green and dark red in the other half-field. The half-field with the greater magnitude of luminance contrast appears to flicker more [(a) and (c)], and flicker appears equal only when both half-fields have the same magnitude of luminance contrast [and thus the same mean luminances for the red and green bars (b); note that the overall mean luminance of the display remains constant]. The equiluminance balance can be estimated by keeping the difference in contrast between the two half-fields constant ( 0.06 in this example) and then covarying the contrasts in both fields, as illustrated in (a)-(c) for successive steps of 0.005 . In the actual experiments the higher contrast was randomly assigned on each trial to either the top or the bottom half-field.

judgments only on the salience of the luminance component (regardless of the correlation between luminance and color). We therefore developed a procedure that required subjects to judge the relative flicker in the stimuli rather than the point of minimum flicker. A fixed difference in luminance contrast was introduced between the top and 


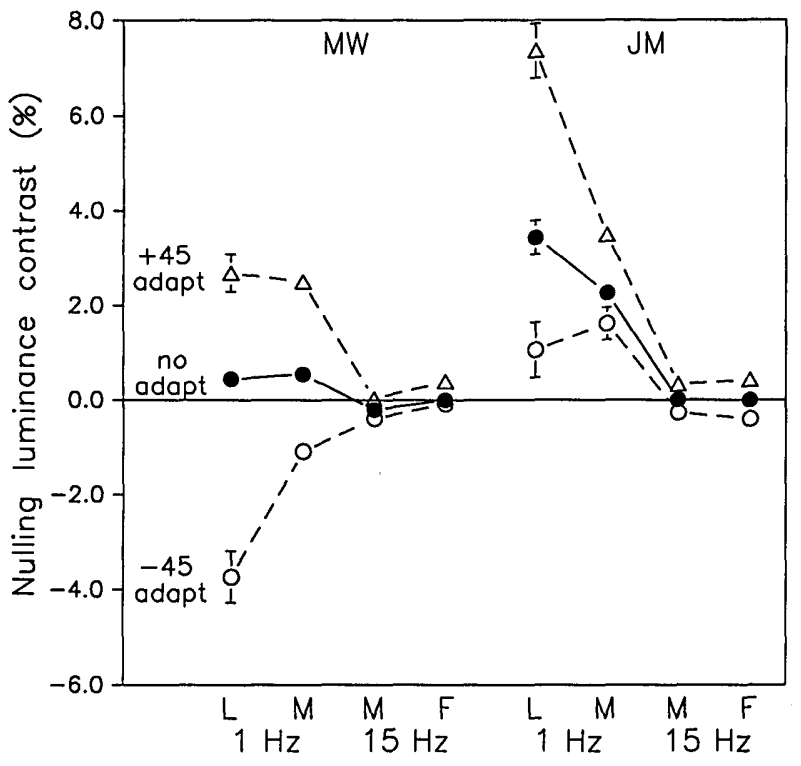

Fig. 3. Equiluminance settings for both observers based on judging lightness (L), motion (M), or flicker (F) in L-M chromatic gratings. Points are the means of eight preadapt or four postadapt settings; error bar, tstandard error. All settings are relative to the preadapt flicker settings (arbitrarily defined as zero luminance contrast). Positive contrasts indicate +luminance contrast added in phase with the $+\mathrm{L}(-\mathrm{M})$ bars.

bottom halves of the field, and luminance contrast of the two half-fields was covaried while observers judged which half-field flickered more. For example, Fig. 2 illustrates a case in which the two half-fields have a fixed contrast difference of 0.06 and covary in steps of 0.005 , so that the two contrasts for comparison vary from $-0.035,+0.025$ [Fig. 2(a)] to $-0.03,+0.03$ [Fig. 2(b)] to $-0.025,+0.035$ [Fig. 2(c)]. The equiluminance point should then occur when the two half-fields have luminance contrast of the same magnitude but combined with the chromatic grating in opposite phases [Fig. 2(b)]. This procedure permitted us to use the same staircase procedure for flicker as for the lightness and minimum-motion judgments and was found to yield highly sensitive estimates of flickerphotometric equivalence even when the point of minimum flicker was poorly defined (e.g., because of losses in luminance contrast sensitivity resulting from the adaptation).
The contrast difference between the half-fields was between 0.04 and 0.16 and was chosen to provide comparable flicker discrimination for different conditions, although estimated equiluminance points did not depend significantly on the magnitude of the difference.

Adapting stimuli were $1.5-\mathrm{c} / \mathrm{deg}$ horizontal gratings formed by combining a luminance and a chromatic grating in one of two phases (see Fig. 1). The two alternative adaptation gratings were thus based on the same luminance and chromatic components but had correlations of opposite sign between luminance and chromaticity. The contrast of the chromatic component equaled the chromatic contrast of the test grating (i.e., $50 \times$ the $1-\mathrm{Hz}$ threshold contrast for $\mathrm{L}-\mathrm{M}$ gratings and $30 \times$ for $\mathrm{S}$ gratings). The luminance component had an equal multiple of the threshold contrast (i.e., $50 \times$ or $30 \times$ the 0.0065 Michelson contrast threshold for detecting a $1-\mathrm{Hz}$ counterphasing luminance grating). The composite contrasts were thus defined by vectors of \pm 45 deg within the luminance-chromatic plane (scaled for the thresholds for the $1-\mathrm{Hz}$ gratings). The spatial phase of the adapting grating was randomly changed on every frame $(60 \mathrm{~Hz})$. This produced a broad temporal-frequency spectrum at any point with no directional bias and minimized differences in the time-averaged chromaticity and luminance at different points in the field.

In daily sessions the four different sensitivity settings (lightness, flicker, and low- and high-frequency motion) were measured before and then after adaptation to one adapting axis, with the order of different measures and adapting axes counterbalanced across sessions. Settings were made by varying the luminance contrast in the test gratings in four randomly interleaved staircases (ten reversals each) with a fixed step size of 0.005 . In adaptation conditions the observers initially adapted for $180 \mathrm{~s}$. One-second test trials were then interleaved with 6-s readaptation intervals (with zero-contrast gaps of $0.5 \mathrm{~s}$ between adaptation and test intervals).

\section{RESULTS}

Figure 3 shows the different equiluminance settings that were made for the $\mathrm{L}-\mathrm{M}$ chromatic gratings before and after adaptation. Adaptation biased both the lightness

Table 2. Three-Way ANOVA of Changes in Equiluminance Settings (Preadapt-Postadapt) for L-M Test and Adaptation Gratings ${ }^{a}$

\begin{tabular}{|c|c|c|c|c|c|c|c|c|}
\hline \multirow{2}{*}{$\begin{array}{l}\text { Source of } \\
\text { Variance }\end{array}$} & \multicolumn{4}{|c|}{ Observer MW } & \multicolumn{4}{|c|}{ Observer JM } \\
\hline & $\mathrm{df}$ & M.S. & $\mathrm{F}$ & $P$ & $\mathrm{df}$ & M.S. & $\mathrm{F}$ & $\mathbf{P}$ \\
\hline $\begin{array}{l}\text { Task (motion versus } \\
\text { flicker/lightness) }\end{array}$ & 1 & 1.96 & 5.04 & 0.03 & 1 & 0.70 & 2.58 & NS \\
\hline $\begin{array}{l}\text { Temporal frequency } \\
\text { (1 versus } 15 \mathrm{~Hz} \text { ) }\end{array}$ & 1 & 1.89 & 4.87 & 0.04 & 1 & 2.24 & 8.27 & 0.008 \\
\hline Task $\times$ frequency & 1 & 3.22 & 8.30 & 0.008 & 1 & 0.76 & 2.82 & NS \\
\hline Task $\times$ adapt & 1 & 3.64 & 9.38 & 0.005 & 1 & 13.26 & 49.06 & 0.0001 \\
\hline Frequency $\times$ adapt & 1 & 46.01 & 118.63 & 0.0001 & 1 & 27.79 & 102.81 & 0.0001 \\
\hline $\begin{array}{l}\text { Task } \times \text { frequency } \\
\quad \times \text { adapt }\end{array}$ & 1 & 2.73 & 7.04 & 0.01 & 1 & 15.10 & 55.85 & 0.0001 \\
\hline Error & 24 & 0.39 & & & 24 & 0.27 & & \\
\hline
\end{tabular}




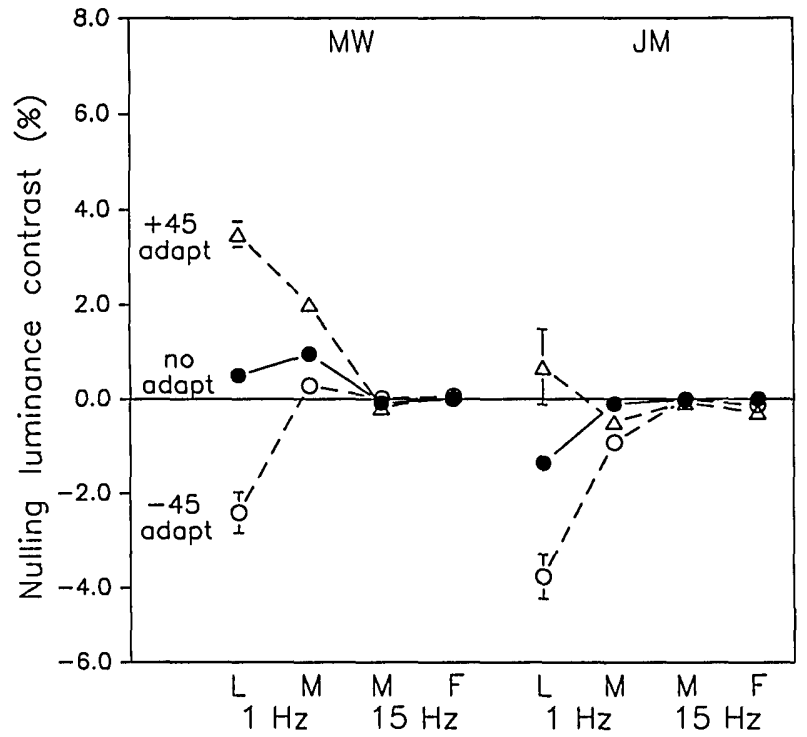

Fig. 4. Equiluminance settings for $\mathrm{S}$ gratings. (Labels are as defined in Fig. 3.)

and the motion settings at $1 \mathrm{~Hz}$ by distorting the relative luminosities of the two colors away from the axis of the adapting modulation (see Fig. 1). For example, after adaptation to the grating defined by the +45 -deg axis (bright red/dark green), the effective luminosity of the red $(+\mathrm{L})$ bars was reduced relative to that of the green $(+M)$ bars. Thus more luminance had to be added to the red bars both to match the green bars for lightness and to null the motion. Conversely, adaptation to the $-45-\mathrm{deg}$ axis induced the opposite biases. However, with one exception ( +45 adapt, observer $\mathrm{MW}$ ), the motion nulls changed less than would be expected from the induced lightness differences. Thus the two tasks depend on processes with different underlying sensitivities, sensitivities that become dissociated with adaptation.

In contrast to these results, adaptation affected the luminance settings for $15-\mathrm{Hz}$ motion or flicker much less (although changes were still significant for JM). This was not because of a lack of adaptation (flicker detection thresholds were substantially elevated); it was because this adaptation did not alter the relative luminosities of the different colors. Consequently, these results dissociate a third, high-temporal-frequency measure of spectral sensitivity that is much less susceptible to biases by contrast adaptation. (Changes in the unadapted minimummotion settings with temporal frequency, as evident in JM's results, were noted by Cavanagh et $a l .^{10}$. See also Ref. 25.)

We assessed these aftereffects by analyzing the results for the two observers in separate three-way ANOVA tests [adapting axis $\times$ temporal frequency $\times$ task (lightness/ flicker versus motion)] with the use of the intrasession differences between preadaptation and postadaptation settings as the dependent variable (Table 2). For both observers the adapting-axis main effect and the interactions that included the adapting axis were significant, thus confirming that luminance settings were biased by adaptation, and the magnitude of this bias depended on both the temporal frequency and the task used to judge the luminosities. Significant task (observer MW) and temporalfrequency ( $\mathrm{MW}$ and $\mathrm{JM}$ ) effects, since they are based on pooling the two oppositely signed adaptation effects, suggest that the two adapting axes produced asymmetric effects for some conditions.

Figure 4 shows measurements for test and adapting gratings whose chromatic contrasts instead modulated only the $\mathrm{S}$ cones (with corresponding ANOVA's in Table 3). In many tasks, including those based on flicker and motion detection, the $\mathrm{S}$ cones appear to contribute only weakly to luminance sensitivity. ${ }^{10,26}$ Our results suggest that signals from the $\mathrm{S}$ cones can interact with luminance signals to bias motion or lightness matches at low temporal frequencies. These biases were again weaker for the motion settings (and consistent only for the -45 adapting axis for JM) and were absent for motion or flicker measurements at $15 \mathrm{~Hz}$.

The fact that adaptation induced little bias in spectral sensitivities at $15 \mathrm{~Hz}$ (but strongly affected luminance contrast sensitivity at this frequency) argues against conespecific light adaptation as the basis for the biases at low temporal frequencies. A cone-specific adaptation effect might also be expected to show little selectivity for the spatial configuration of the stimuli. To test this we compared the $1-\mathrm{Hz}$ motion and lightness biases after adapta-

Table 3. Three-way ANOVA of Changes in Equiluminance Settings (Preadapt-Postadapt) for $\mathbf{S}$ Test and Adaptation Gratings ${ }^{a}$

\begin{tabular}{|c|c|c|c|c|c|c|c|c|}
\hline \multirow{2}{*}{$\begin{array}{c}\text { Source of } \\
\text { Variance }\end{array}$} & \multicolumn{4}{|c|}{ Observer MW } & \multicolumn{4}{|c|}{ Observer JM } \\
\hline & $\mathrm{df}$ & M.S. & $\mathrm{F}$ & $\mathrm{P}$ & $\mathrm{df}$ & M.S. & $\mathrm{F}$ & $P$ \\
\hline $\begin{array}{l}\text { Task (motion versus } \\
\text { flicker/lightness) }\end{array}$ & 1 & 0.10 & 0.83 & NS & 1 & 0.16 & 0.27 & NS \\
\hline $\begin{array}{l}\text { Temporal frequency } \\
\text { (1 versus } 15 \mathrm{~Hz})\end{array}$ & 1 & 0.002 & 0.02 & NS & 1 & 0.54 & 0.91 & NS \\
\hline Task $\times$ frequency & 1 & 0.06 & 0.52 & NS & 1 & 1.04 & 1.78 & NS \\
\hline $\begin{array}{l}\text { Adaptation axis } \\
\quad(+45 \mathrm{deg} \text { versus }-45 \mathrm{deg})\end{array}$ & 1 & 32.40 & 284.58 & 0.0001 & 1 & 13.06 & 22.27 & 0.0001 \\
\hline Task $\times$ adapt & 1 & 9.22 & 81.01 & 0.0001 & 1 & 4.78 & 8.14 & 0.009 \\
\hline Frequency $\times$ adapt & 1 & 31.28 & 274.77 & 0.0001 & 1 & 11.64 & 19.85 & 0.0002 \\
\hline $\begin{array}{l}\text { Task } \times \text { frequency } \\
\quad \times \text { adapt }\end{array}$ & 1 & 9.79 & 85.99 & 0.0001 & 1 & 7.90 & 13.47 & 0.001 \\
\hline Error & 24 & 0.11 & & & 24 & 0.59 & & \\
\hline
\end{tabular}

${ }^{a}$ Abbreviations as in Table 2. 


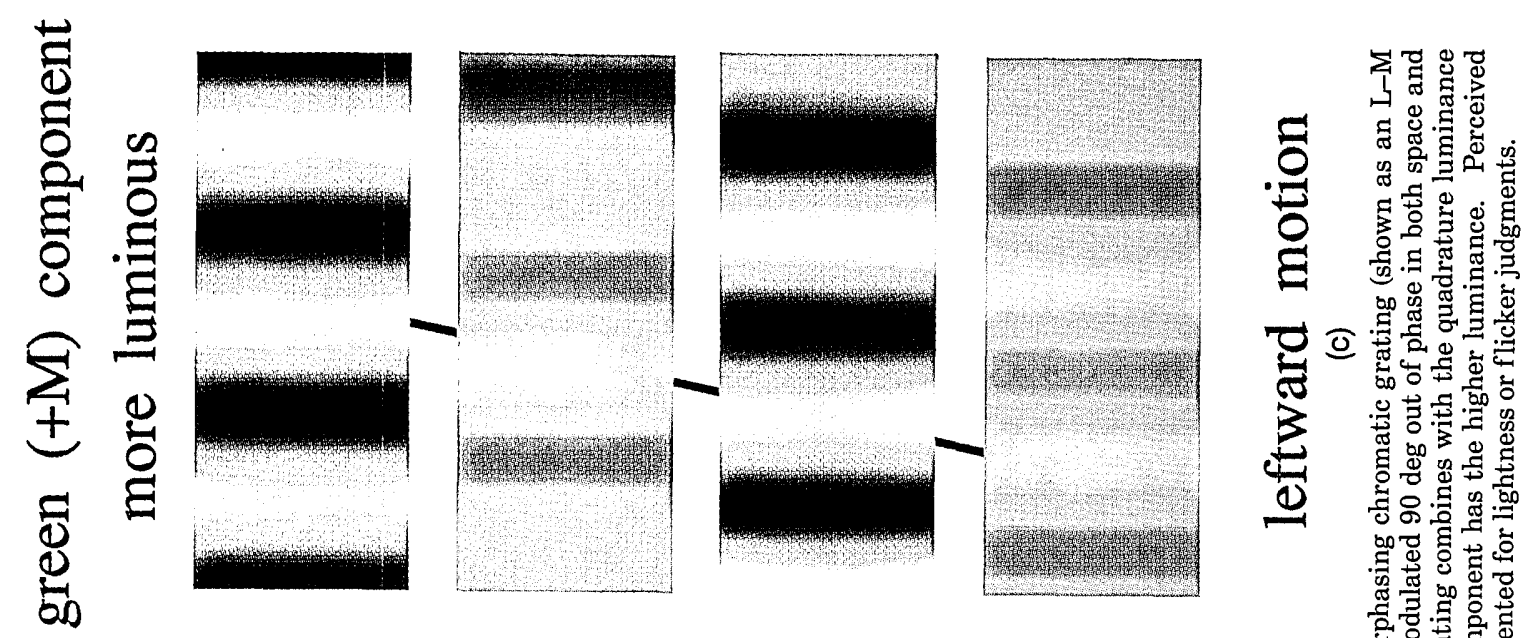

ত。

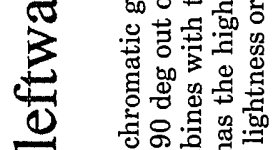

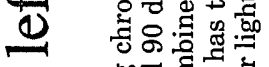

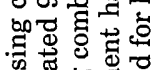

究

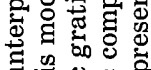

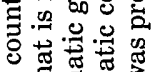

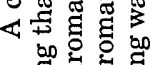
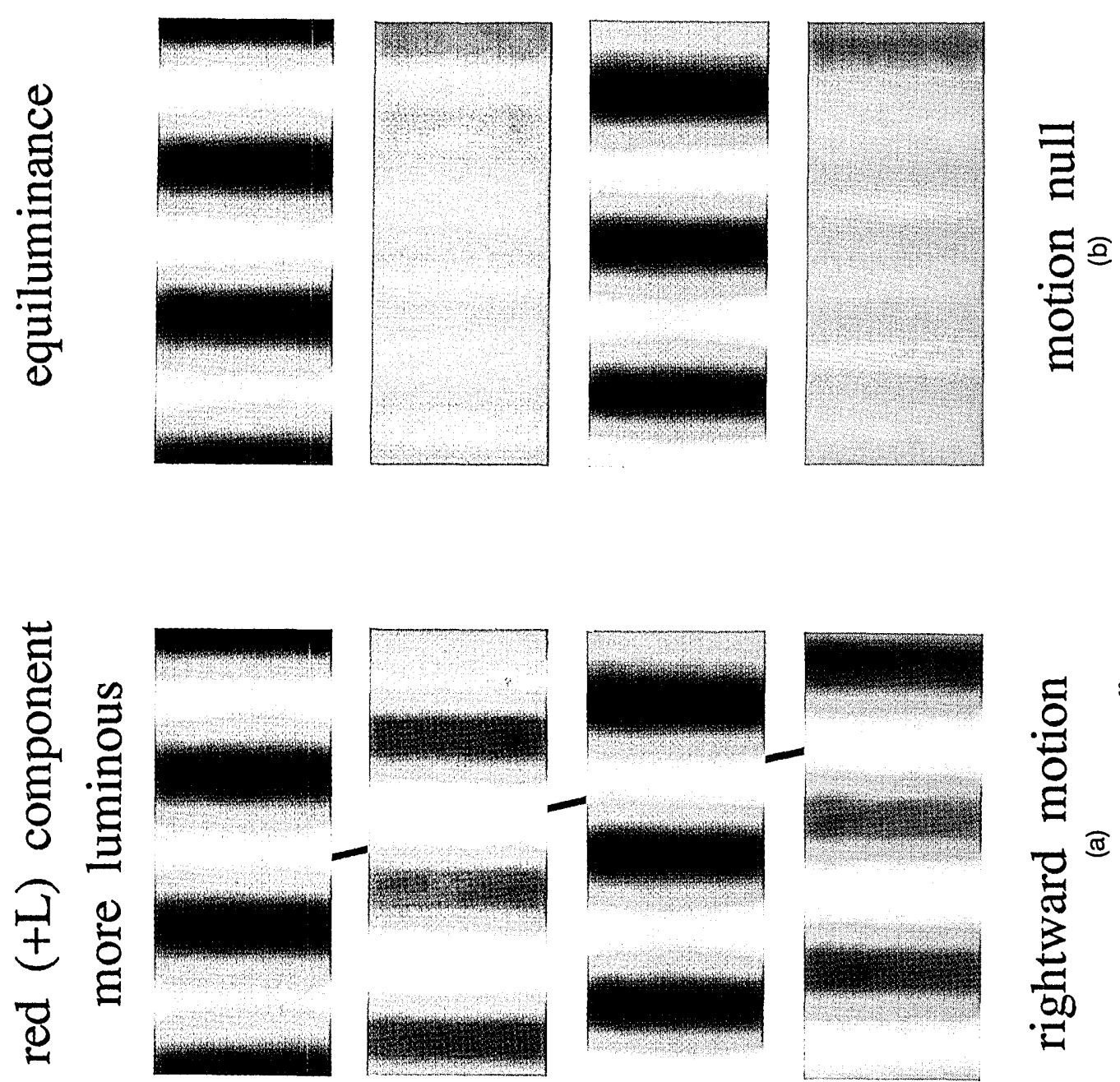

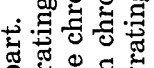



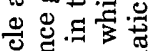

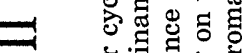

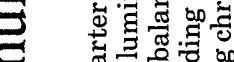

궁

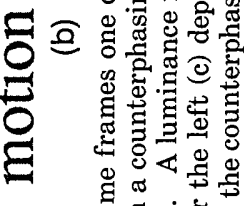

零的园

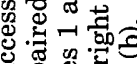

吅

का

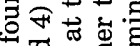

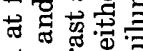

실ㅇㅇㅇ

为
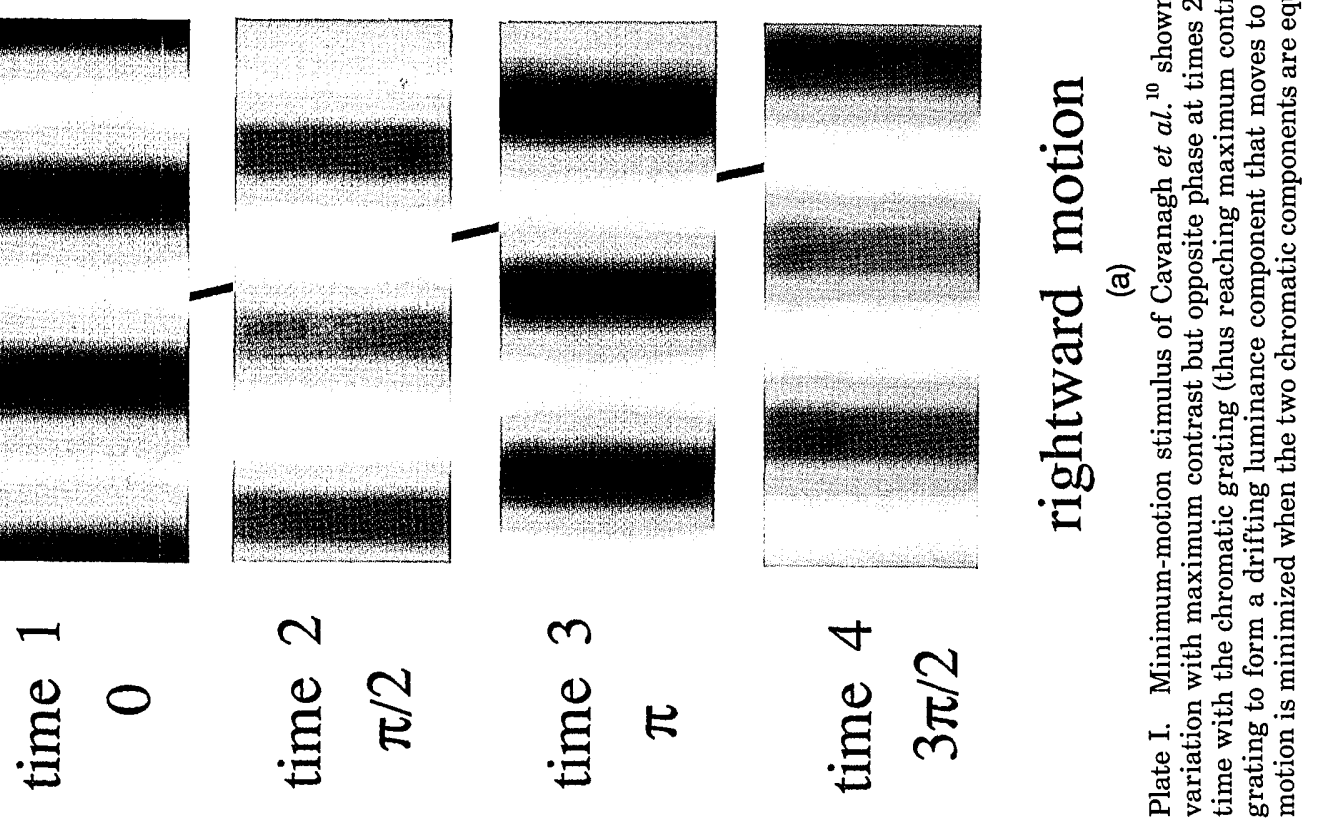


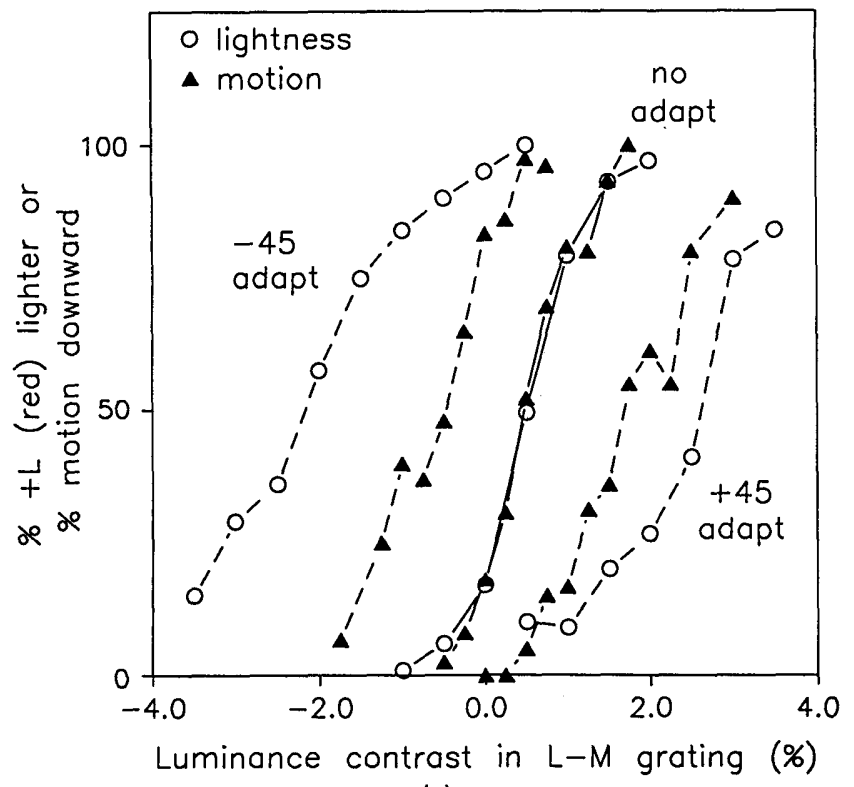

(a)

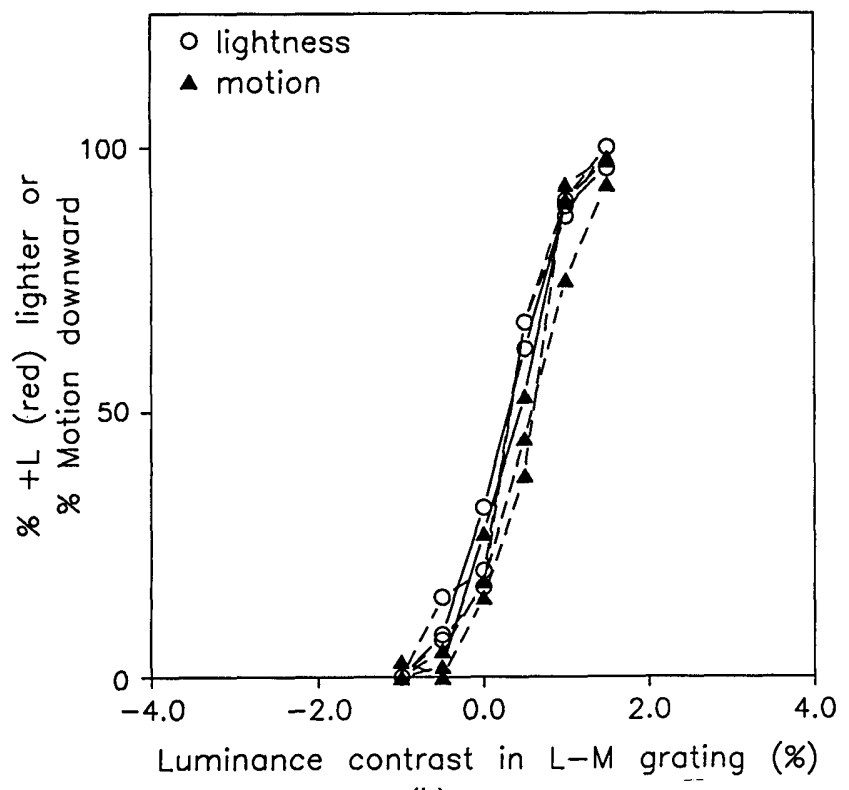

(b)

Fig. 5. (a) Psychometric functions for judging motion or lightness in $1-\mathrm{Hz} \mathrm{L}-\mathrm{M}$ gratings before or after adaptation to gratings. (b) Lightness and motion settings after adaptation to a uniform 2-deg field with the same temporal contrast as the adapting grating of $5 \mathrm{~A}$.

tion to either the adapting grating or a uniform 2-deg field that had the same temporal contrast as the adapting grating. In these measurements, which were performed before we adopted the final conditions described above, we estimated luminosities with a method of constant stimuli and we weighted the adapting stimulus toward lower temporal frequencies [by waiting a random number of frames (between 1 and 30 ) before a new random phase was selected]. Figure 5 shows that the biases in lightness and motion settings after grating adaptation [Fig. 5(a)] were absent after uniform-field adaptation [Fig. 5(b)], indicating that the aftereffects are spatially selective.

In the minimum-motion stimulus, adaptation could influence the settings not only by changing luminosities in the chromatic grating but also by inducing color differ- ences between the bright and dark bars of the added luminance grating [Fig. 6(a)]. This induced chromatic contrast could combine with the chromatic test grating to induce chromatic motion in the same direction as induced luminance motion. We therefore performed the converse experiment of varying chromatic contrast in the luminance grating either to null perceived color differences between the light and dark bars of the luminance grating or to null perceived motion in the grating pair. In this case adaptation altered color-motion nulls substantially more than would be predicted from the induced color change in the luminance grating, the opposite of the dissociations we found for luminance contrast [Fig. 6(b)]. This could occur if adaptation induces both luminance and chromatic changes in the gratings, but chromatic contrast is much weaker than luminance contrast at supporting or nulling the resulting motion. Confirming this, we found that when opposing directions of luminance and chromatic motion $^{27}$ are introduced into the stimulus the perceived direction is strongly dominated by the luminance component. ${ }^{28}$ Thus the changes that adaptation induces in the motion settings are primarily the result of how it affects the luminosities of the patterns.

\section{DISCUSSION}

Many studies have used stimuli of varying color but constant luminance to try to isolate chromatic pathways (e.g., Ref. 4). Our results show that different operational measures for equating luminosities yield different estimates, depending on the adapted state of the observer. These dissociations apparently occur because the channels mediating both motion and lightness judgments at $1 \mathrm{~Hz}$ do not represent luminance and chromatic contrast independently. Instead, the channels exhibit sensitivity changes that are selective for how luminance and chromatic contrast are combined in the adapting stimulus. The loss of these luminance-chromatic interactions at $15 \mathrm{~Hz}$ presumably occurs because at these frequencies sensitivity to chromatic contrast is much lower; if at these frequencies the visual system is primarily sensitive only to the luminance variations in the stimulus, then it would not be expected to show aftereffects that depend on how luminance and color in the stimuli are covarying. This selective loss in chromatic sensitivity could result either because a different subset of channels encodes the high-temporalfrequency patterns or because the channels change their tuning properties at higher temporal frequencies, for example, by losing color opponency. ${ }^{25,29}$ An absence of adaptation-dependent interactions between luminance and color has also been reported in a threshold-detection task (measured for $4-\mathrm{Hz}$ test stimuli). ${ }^{6}$

Our results support several previous studies in suggesting that chromatic contrast contributes to motion perception. This contribution was suggested as early as 1911 by Wohlgemuth, who demonstrated a motion aftereffect at equiluminance. ${ }^{30}$ Moreover, the fact that adaptation induces weaker biases in luminance minimum-motion settings than in lightness matches and induces stronger biases in chromatic minimum motion than in chromatic matches suggests that chromatic contrast provides a weaker signal in motion perception than in color appearance. This property has been suggested by the large variety of impairments in motion perception that are observed 


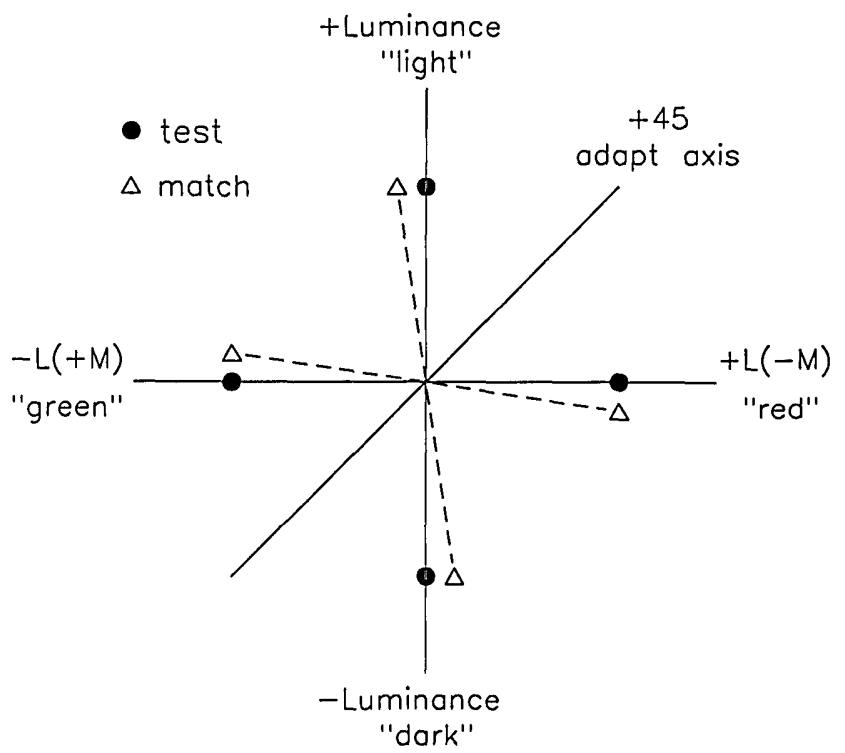

(a)

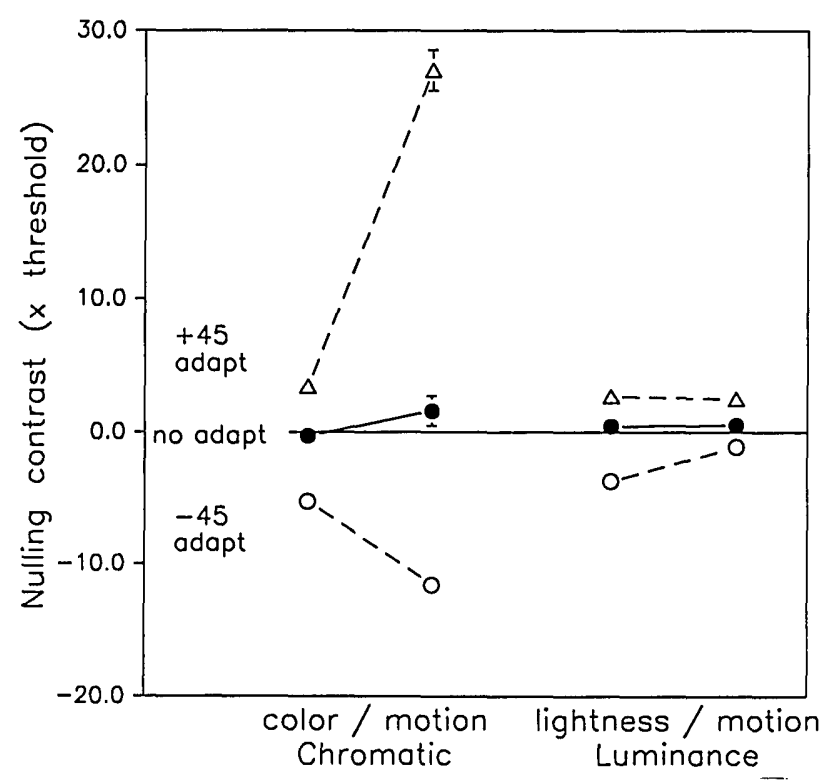

(b)

at equiluminance. ${ }^{31}$ In a neutral state of adaptation, luminance and chromatic contrast appear to be represented as orthogonal dimensions, and it is this orthogonality that forms the logical basis for the minimum-motion technique. However, the biases we find in low-frequency minimummotion settings suggest that these stimulus dimensions are not encoded as orthogonal after adaptation. The induced luminance contrasts we observed of 0.01-0.02 could provide a potent motion signal because many aspects of motion perception appear to saturate at low luminance contrasts (see, e.g., Ref. 32).

The perceptual biases that adaptation induces in the axes of color space are analogous to spatial-adaptation phenomena such as the tilt aftereffect for the perceived orientation of lines. ${ }^{33}$ Such aftereffects are commonly explained by assuming that the test stimulus is repre-

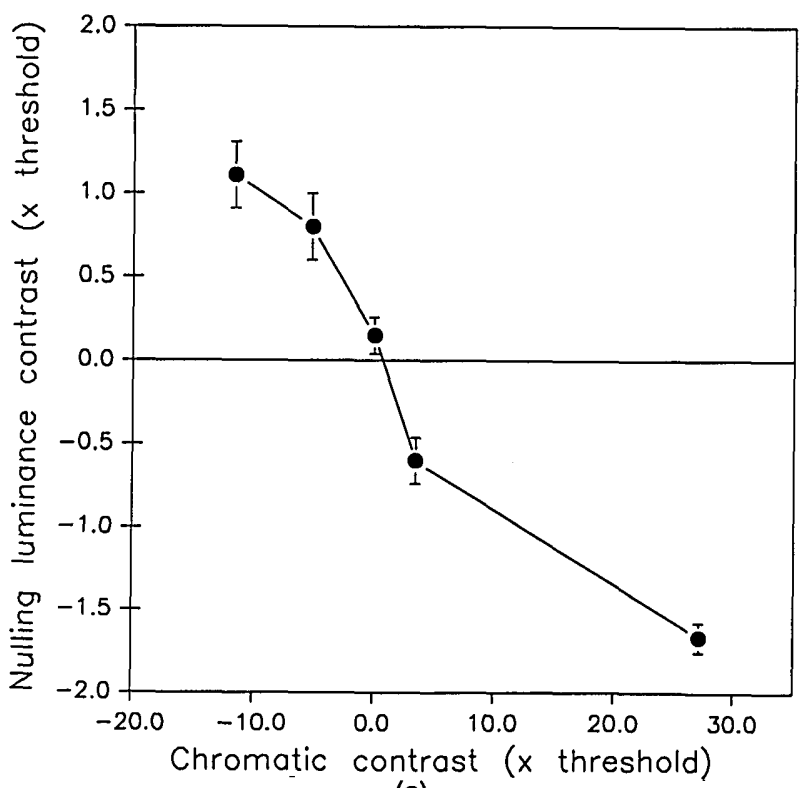

(c)

Fig. 6. (a) Adaptation not only induces lightness differences in a chromatic grating but can also induce color differences between the light and dark bars of a luminance grating, again tilting the axis that defines the grating away from the adapting axis. ${ }^{7}$ The phase relationship between the apparent luminance and the apparent chromatic variations should be the same in both gratings (e.g., both gratings should appear to vary between light green and dark red). Consequently, when both gratings are presented in the minimum-motion stimulus, the induced luminance and chromatic contrasts should both drift in the same direction. If the two gratings instead have different relationships between luminance and chromatic contrast (e.g., if one grating varies between light green and dark red while the second varies between light red and dark green), then the moving luminance and chromatic components will instead drift in opposite directions [as in the experiment of (c)]. (b) L-M chromatic contrast that is required to null perceived color differences in the luminance grating or perceived motion $(1 \mathrm{~Hz})$. Points plot the settings either before or after adaptation to luminance-chromatic gratings of the same contrast as in Fig. 3. Luminance settings are replotted from Fig. 3 (observer MW) for comparison. Positive values indicate that $+\mathrm{L}(-\mathrm{M})$ contrast was added to the +luminance bars of the grating. (c) Minimum-motion settings for grating pairs that give rise to opposing directions of luminance and chromatic motion (without adaptation). Points indicate the amount of luminance contrast that had to be added to the $50 \times$ threshold chromatic grating to null perceived motion. The counterphasing reference grating had a fixed luminance contrast ( $21 \times$ threshold) and varying amounts of chromatic contrast (indicated by the abscissa) that equaled the chromatic null points of (b).

sented by a distribution of activity across many labeled channels, each of which is tuned to a different stimulus value (e.g., different orientation), and that adaptation distorts this representation by selectively desensitizing a subpopulation of the channels. ${ }^{34}$ Our results could similarly reflect the intrinsic variability in the weights of cone inputs to different neurons as observed physiologically, and the different biases we observe are consistent with different degrees of variability within the neural populations that underlie different perceptual tasks. For example, motion and flicker perception have been associated with magnocellular pathways, whereas lightness and color appearance have instead been linked to parvocellular pathways. ${ }^{4,35}$ Within both subsystems individual neurons vary with respect to the plane in color-luminance space that gives a null response, and they correspondingly vary 
in the combination of luminance and chromatic contrast to which they are optimally tuned. Yet this variability is substantially greater among parvocellular cells, which exhibit strong color opponency, than among magnocellular units, whose preferred directions cluster close to the luminance axis (see, e.g., Ref. 23, Figs. 5A and 13A, and Ref. 36, Fig. 4). Adaptation to particular orientations within color-luminance space could therefore produce larger distortions within the parvocellular distribution and could thus induce larger distortions in judgments of luminous efficiency that depend on this distribution. ${ }^{37}$ However, the site of the sensitivity changes is likely to be cortical, since geniculate cells exhibit little adaptation to contrast $^{23,38}$ and do not show the pronounced losses in chromatic sensitivity at high temporal frequencies that are suggested by psychophysical measures. ${ }^{23,39}$

Barlow and Földiák $\mathrm{k}^{40}$ proposed an additional form of contrast adaptation based on mutual inhibition between channels whenever their outputs are correlated: the inhibition serves to alter the channels' tuning functions in such a way as to decorrelate their outputs. We have noted previously that such a process could simulate the presence of an adaptable channel that is specific for any direction in color-luminance space. ${ }^{7}$ For example, phase-specific inhibition between luminance and chromatic mechanisms could bias the preferred axes of both mechanisms away from the adapting axis (since a stimulus that originally isolated the chromatic mechanism would now also induce a response of opposite sign in the luminance mechanism and vice versa). This adaptive realignment could thus provide an alternative basis for the perceptual distortions that we observe, even if color and luminance are encoded by only a small number of discrete channels. ${ }^{7}$ But if the interactions postulated by Barlow and Földiák occur, our present results suggest that they do not uniformly recalibrate spectral sensitivity within different pathways.

\section{ACKNOWLEDGMENTS}

We are grateful to C. F. Stromeyer III for comments on an earlier draft of this manuscript and to the Wellcome Trust for supporting the publication of Plate I. This research was supported by the UK Science and Engineering Research Council, the Isaac Newton Trust, and a National Institutes of Health fellowship granted to M. Webster.

\section{REFERENCES AND NOTES}

1. P. Lennie and M. D'Zmura, "Mechanisms of color vision," CRC Crit. Rev. Neurobiol. 3, 333-400 (1988).

2. V. C. Smith and J. Pokorny, "Is there a luminance channel?" Farbe 34, 123-128 (1987).

3. S. Exner, Entwurf zu einer physiologischen Erklärung der psychischen Erscheinungen (Deuticke, Leipzig, 1894); S. M. Zeki, "Functional specialisation in the cortex of the rhesus monkey," Nature (London) 274, 423-428 (1978); R. Shapley, "Visual sensitivity and parallel retinocortical channels," Ann. Rev. Psychol. 41, 635-658 (1990).

4. M. S. Livingstone and D. H. Hubel, "Segregation of form, color, movement, and depth: anatomy, physiology, and perception," Science 240, 740-749 (1988).

5. C. Blakemore and F. W. Campbell, "On the existence of neurones in the human visual system selectively sensitive to the orientation and size of retinal images," J. Physiol. (London) 203, 237-260 (1969); T. Benzschawel and S. L. Guth, "Post- receptor chromatic mechanisms revealed by flickering vs fused adaptation," Vision Res. 22, 66-75 (1982); A. Bradley, E. Switkes, and K. K. De Valois, "Orientation and spatial frequency selectivity of adaptation to color and luminance gratings," Vision Res. 28, 841-856 (1988).

6. J. Krauskopf, D. R. Williams, and D. W. Heeley, "Cardinal directions of color space," Vision Res. 22, 1123-1131 (1982).

7. M. A. Webster and J. D. Mollon, "Changes in colour appearance following post-receptoral adaptation," Nature (London) 348, 235-238 (1991).

8. Lightness refers to the achromatic quality (apparent reflectance) of objects that are perceived as not self-luminous, whereas brightness refers to the quality of objects that are perceived as self-luminous. In the case of an isolated spot displayed on an otherwise dark CRT display, the appropriate term would be "brightness," whereas lightnesses can be simulated on a CRT if the scene is spatially complex and includes sufficient gray scale. Gratings such as those we used represent an intermediate stimulus. We use "lightness" because a gray or dark quality is induced in the less luminous bars and provides a salient cue for the observer.

9. O. N. Rood, "On a flicker photometer," Science 7, 757-759 (1898).

10. P. Cavanagh, D. I. A. MacLeod, and S. M. Anstis, "Equiluminance: spatial and temporal factors and the contribution of blue-sensitive cones," J. Opt. Soc. Am. A 4, 1428-1438 (1987).

11. C. F. Stromeyer III, R. E. Kronauer, J. C. Madsen, and S. A. Klein, "Opponent-movement mechanisms in human vision," J. Opt. Soc. Am. A 1, 876-884 (1984).

12. S. M. Anstis and P. Cavanagh, "A minimum motion technique for judging equiluminance," in Colour Vision: Physiology and Psychophysics, J. D. Mollon and L. T. Sharpe, eds. (Academic, London, 1983), pp. 156-166.

13. P. K. Kaiser, "Sensation luminance: a new name to distinguish CIE luminance from luminance dependent on an individual's spectral sensitivity," Vision Res. 28, 455-456 (1988).

14. Commission Internationale de l'Eclairage, Light as a True Visual Quantity: Principles of Measurement, Publication No. 41 (TC-1.4) (Bureau Central CIE, Paris, 1978).

15. A. Kohlrausch, "Über den Helligkeitsvergleich verschiedener Farben," Pfluegers Arch. Gesamte Physiol. Menschen Tiere 200, 210-215 (1923); G. Wagner and R. M. Boynton, "Comparison of four methods of heterochromatic photometry," J. Opt. Soc. Am. 62, 1508-1515 (1972).

16. S. L. Guth, N. J. Donley, and R. T. Marroco, "On luminance additivity and related topics," Vision Res. 9, 537-575 (1969).

17. Weak additivity failures in minimum-motion settings have been reported by P. K. Kaiser, R. L. P. Vimal, W. B. Cowan, and $\mathrm{H}$. Hibino, "Nulling of apparent motion as a method for assessing sensation luminance: an additivity test," Color Res. Appl. 14, 187-191 (1989).

18. The modern version of this hypothesis was anticipated in 1923 by A. Kohlrausch ("Theoretisches und Praktisches zur heterochromen Photometrie," Pfluegers Arch. Gesamte Physiol. Menschen Tiere 200, 216-220), who distinguished clearly between the spectral sensitivity curve that was obtained from flicker photometry or acuity measurements and the broader curve that was obtained from direct heterochromatic brightness matching. He wrote: "this extra efficiency (Wirksamkeitplus) of the upper curve can be traced to the characteristic luminosities of individual colorsthe attribute termed 'chromatic glow' (Farbenglut) by von Helmholtz. The chromatic glow is particularly conspicuous to a color-normal when various very saturated colors are juxtaposed to white. It increases from yellow towards both ends of the spectrum. Accordingly the area between the two trichromatic photopic spectral sensitivity curves would be the graphical expression of the varying 'glow' of colors, which under some circumstances asserts itself in addition to the actual luminance (Helligkeit). On this hypothesis, what remains striking, and worth following up, is that the chromatic glow strongly influences some discriminations and does not at all influence spatial and temporal discriminations." The reference to von Helmholtz is to $\mathrm{H}$. von Helmholtz, Handbuch der physiologischen Optik, 2nd ed. (Voss, Hamburg, 1896), p. 347.

19. This need not reflect a rigid rotation of the equiluminant 
plane because the magnitude of the tilt may depend on the chromatic contrast.

20. D. Jameson and L. M. Hurvich, "Color adaptation: sensitivity, contrast, after-images," in Handbook of Sensory Physiology, D. Jameson and L. M. Hurvich, eds. (SpringerVerlag, New York, 1972) Vol. 7, Part 4, pp. 568-581.

21. M. A. Webster, K. K. De Valois, and E. Switkes, "Orientation and spatial-frequency discrimination for luminance and chromatic gratings," J. Opt. Soc. Am. A 7, 1034-1049 (1990).

22. Y. Le Grand, "Les seuils différentials de couleurs dans la théorie de Young," Rev. Opt. Théor. Instrum. 28, 261-278 (1949); J. D. Mollon, "Tho' she kneel'd in that Place where they grew ...," J. Exp. Biol. 146, 21-38 (1989).

23. A. M. Derrington, J. Krauskopf, and P. Lennie, "Chromatic mechanisms in lateral geniculate nucleus of macaque," J. Physiol. (London) 357, 241-265 (1984).

24. D. I. A. MacLeod and R. M. Boynton, "Chromaticity diagram showing cone excitation by stimuli of equal luminance," J. Opt. Soc. Am. 69, 1183-1186 (1979).

25. D. H. Kelly, "Spatiotemporal variation of chromatic and achromatic contrast thresholds," J. Opt. Soc. Am. 73, 742750 (1983).

26. P. Whittle, "The brightness of coloured flashes on backgrounds of various colours and luminances," Vision Res. 13, 621-638 (1973); V. C. Smith and J. Pokorny, "Spectral sensitivity of the foveal cone pigments between 400 and $500 \mathrm{~nm}$," Vision Res. 15, 161-171 (1975); A. Eisner and D. I. A. MacLeod, "Blue-sensitive cones do not contribute to luminance," J. Opt. Soc. Am. 70, 121-123 (1980); A. Stockman, D. I. A. MacLeod, and D. D. DePriest, "The temporal properties of the human short-wave photoreceptors and their associated pathways," Vision Res. 31, 189-208 (1991); J. Lee and C. F. Stromeyer III, "Contribution of human short wave cones to luminance and motion detection," J. Physiol. (London) 413, 563-593 (1989)

27. P. Cavanagh and S. Anstis, "The contribution of color to motion in normal and color-deficient observers," Vision Res. 31, 2109-2148 (1991)

28. Changes in the minimum-motion nulls as the angle defining the reference grating is varied have also been reported by C. F. Stromeyer III, R. T. Eskew, and R. E. Kronauer, "Equiluminance and minimal motion," in Annual Meeting, Vol. 15 of 1990 Technical Digest Series (Optical Society of America, Washington, D.C., 1990), p. 183.

29. P. Gouras and E. Zrenner, "Enhancement of luminance flicker by color-opponent mechanisms," Science 205, 587589 (1979); C. R. Ingling and E. Martinez-Uriegas, "The spatiotemporal properties of the $\mathrm{r}-\mathrm{g} x$-cell channel," Vision Res. 25, 33-38 (1985).

30. A. Wohlgemuth, "On the after-effect of seen movement," Br. J. Psychol. Monograph Suppl. 1, 1-117 (1911).

31. S. M. Anstis, "Phi movement as a subtraction process," Vision Res. 10, 1411-1430 (1970); V. S. Ramachandran and R. L. Gregory, "Does colour provide an input to human motion perception?" Nature (London) 275, 55-56 (1978); J. D. Moreland, "Spectral sensitivity measured by motion photometry," in Colour Vision Deficiencies V, G. Verriest, ed. (Hilger, Bristol, UK, 1980), pp. 299-305; P. Cavanagh, C. W. Tyler, and O. E. Favreau, "Perceived velocity of moving chromatic gratings," J. Opt. Soc. Am. A 1, 893-899 (1984); T. Troscianko, "Perception of random-dot symmetry and apparent movement at and near isoluminance," Vision Res. 27, 547-554 (1987); D. L. Lindsey and D. Y. Teller, "Motion at isoluminance: discrimination/detection ratios for moving isoluminant gratings," Vision Res. 30, 1751-1761 (1990); K. T. Mullen and J. C. Boulton, "Absence of smooth motion perception in color vision," Vision Res. 32, 483-488 (1992); F. L. Kooi and K. K. De Valois, "The role of color in the motion system," Vision Res. 32, 657-668 (1992).

32. L. S. Stone, A. B. Watson, and J. B. Mulligan, "Effect of contrast on the perceived direction of a moving plaid," Vision Res. 30, 1049-1067 (1990).

33. J. J. Gibson and M. Radner, "Adaptation, after-effect and contrast in the perception of tilted lines. I. Quantitative studies," J. Exp. Psychol. 20, 453-467 (1937).

34. N. S. Sutherland, "Figural after-effects and apparent size," Q. J. Exp. Psychol. 13, 222-228 (1961).

35. B. B. Lee, P. R. Martin, and A. Valberg, "The physiological basis of heterochromatic flicker photometry demonstrated in the ganglion cells of the macaque retina," J. Physiol. (London) 404, 323-347 (1988); P. H. Schiller, N. K. Logothetis, and E. R. Charles, "Functions of the colour-opponent and broadband channels of the visual system," Nature (London) $\mathbf{3 4 3}$, 68-70 (1990).

36. N. K. Logothetis, P. H. Schiller, E. R. Charles, and A. C. Hurlbert, "Perceptual deficits and the activity of the coloropponent and broad-band pathways at isoluminance," Science 247, 214-217 (1990).

37. Our measures could be accounted for by the parvocellular and magnocellular distributions in more than one way. For example, the $15-\mathrm{Hz}$ measures might depend on the magnocellular responses, whereas the $1-\mathrm{Hz}$ measures could reflect different weights of magnocellular and parvocellular contributions. Thus we cannot unambiguously associate our specific measures with either pathway.

38. L. Maffei, A. Fiorentini, and S. Bisti, "Neural correlate of perceptual adaptation to gratings," Science 182, 1036-1038 (1973); I. Ohzawa, G. Sclar, and R. D. Freeman, "Contrast gain control in the cat's visual system," J. Neurophysiol. 54, 651-667 (1985).

39. B. B. Lee, P. R. Martin, and A. Valberg, "Sensitivity of macaque retinal ganglion cells to chromatic and luminance flicker," J. Physiol. (London) 414, 223-243 (1989).

40. H. B. Barlow and P. Földiák, "Adaptation and decorrelation in the cortex," in The Computing Neuron, R. M. Durbin, C. Miall, and G. J. Mitchison, eds. (Addison-Wesley, New York, 1989), pp. $54-72$. 